Gefässchirurgie $2020 \cdot 25: 215-216$ https://doi.org/10.1007/s00772-020-00658-9

๑) Springer Medizin Verlag GmbH, ein Teil von Springer Nature 2020

\section{A. Larena-Avellaneda ${ }^{1,2}$}

${ }^{1}$ Klinik und Poliklinik für Gefäßmedizin, Universitäres Herz- und Gefäßzentrum Hamburg, Universitätsklinikum Hamburg-Eppendorf, Hamburg, Deutschland

${ }^{2}$ Abteilung für Gefäß- und endovaskuläre Chirurgie, Asklepios Klinik Altona, Hamburg, Deutschland

\title{
Non vitae sed scientiam discimus. Oder: Warum forschen wir?
}

Geschätzte Leserinnen und Leser,

Sie haben nun eine Ausgabe Ihrer Lieblingsfachzeitschrift mit dem Leitthema "Grundlagenforschung" vor sich liegen. Wir (Schriftleitung, Herausgeber, Hefteditoren) wissen, dass sich die meisten von Ihnen nicht brennend für dieses Thema interessieren. Oder vielleicht haben Sie Interesse, aber der Alltag lässt es nicht $\mathrm{zu}$, dass Sie sich tiefer damit befassen. Dies spiegeln übrigens auch die Sitzungen auf unseren Kongressen wider. In den Sessions zu Forschungsthemen verirren sich - neben den Vorsitzenden und Rednern - leider oft nur sehr wenige $\mathrm{Zu}$ hörer. Immerhin nehmen die dann aber auch rege an den Diskussionen teil. Heute haben Sie nun Gelegenheit, sich hier ganz in Ruhe einzulesen.

In diesem Editorial möchte ich aber die Perspektive der Autoren beleuchten. Auch ich habe Grundlagenforschung betrieben, das ist allerdings schon ein wenig her, und man kann nicht alles dauerhaft auf einem hohen Niveau halten. Eine Frage zur Forschung stelle ich den Studierenden im Rahmen unseres wissenschaftlichen Wahlpflichtcurriculums:

\section{Warum forschen wir?}

Forschung kostet Zeit und Geld. Warum tun wir uns dies an? Als Ärzte? Als Gefäßchirurgen? Die erste Antwort, die mir immer durch den Kopf geht (auf die aber die Studierenden fast nie kommen), ist: Neugier. Wie ist dies so schön

Frei nach Seneca: Non vitae sed scholae discimus („Nicht für das Leben, sondern für die Schule lernen wir"). im Lexikon der Psychologie definiert [1]:

Neugier, Wissensdurst; ein Zustand, der einhergeht mit einer erhöhten Bereitschaft eines Organismus, sich neuen, ungewohnten und komplexen Situationen und $\mathrm{Ob}$ jekten auszusetzen bzw. diese aktiv aufzusuchen (...). Sie sei die Grundlage für die besonderen wissenschaftlichen und kulturellen Leistungen der Menschen.

Vor diesem Hintergrund wird schnell klar, wer der erste neugierige gefäßchirurgische Forscher war: der große Anatom Andreas Vesalius. Im 7. Buch seiner "Fabrica“ findet sich folgender Auszug (-Abb. 1):

Gelb markiert ist klar zu lesen, dass der Forscher die Leiste freigelegt hat und die Arterie längs eröffnete. „Canaliculum ex arundine" ist ein Schilfrohr. Somit hat Vesalius den ersten Bypass im Tierversuch gelegt. Hierbei ist auch folgendes interessant: Zahlreiche Autoren zitieren Vesalius, aber mal sind es Kaninchen, die operiert wurden, mal waren es die Carotiden. Es lohnt sich also immer, bis zur Primärquelle zu gehen, wenn man sicher sein will.

Auch wenn er inzwischen aufgrund seiner dubiosen Gedanken zum Ende seiner Karriere hin in Ungnade gefallen ist. - Ein weiterer großer Forscher war Alexis Carrel, der die moderne Gefäßchirurgie begründet hat. Seine Antriebsgründe waren offensichtliche Hilflosigkeit anderer: 1873 in Lyon geboren, sah er 1894 mit an, wie auf den Präsidenten der Französischen Republik ein Attentat verübt wurde. Die besten Chirurgen Frankreichs waren nicht in der Lage, die Messerstichverletzung der Pfortader zu versorgen.
Carrel war erschüttert, und konzentrierte sich fortan auf die Chirurgie der Gefäße. Allerdings fiel er durch die Aufnahmeprüfung in Frankreich und wanderte nach Amerika aus. Seine wegweisenden Arbeiten verhalfen ihm 1912 zum Nobelpreis für Medizin für seine Arbeiten auf dem Gebiet der Transplantationen und der Gefäßchirurgie.

Nicht ganz so selbstlos ist ein weiterer wesentlicher Antrieb, Forschung als Chirurg zu betreiben: die Belohnung der Forschenden. Eine solche Belohnung kann verschiedene Formen annehmen. Natürlich denkt man sofort an Titel: Die Promotions- und auch Habilitationsleistungen, die zu den begehrten akademischen Titeln führen, beruhen - neben der Lehre - insbesondere auf Forschungsleistungen. $\mathrm{Zu}$ weiteren Belohnungen gehören dann natürlich auch die Sichtbarkeit durch Publikationen und der Besuch von Fachkongressen in anderen Städten und/ oder Ländern. Aber auch Geld muss als Belohnung genannt werden. Damit meine ich an dieser Stelle nicht das höhere Gehalt, das ggf. der Titel mit sich bringt, sondern das Einwerben von Geld. Der Erhalt von großen Beträgen in Drittmitteln ist in vielen Universitäten $\operatorname{der} \mathrm{Maß}$ stab schlechthin. Viele Millionen von unabhängigen Mittelgebern wie der DFG, BMBF oder weiteren sichert einem Forscher bzw. einer Forscherin ein großes Renommee und öffnet Türen für Ordinariate. Leistungsorientierte Mittel (LOM) richten sich nicht nur nach der Qualität und dem Ort von Publikation (hierüber habe ich an dieser Stelle schon oft geschrieben, und leider gibt es für Veröffentlichungen in der Gefässchirurgie nicht viele LOM-Punkte), sondern auch nach 
aut contentam in arterijs materiam pulfuum opificem exiftere, uerùm à corde cam uirtutem pendere, præterquàm quod arteriam unculo interceptam nỏ amplius fub uinculo pulfare cer. nimus, licebit inguinis femoris ue arterix longam fectionem inducere, \& canaliculum ex arun dine tam craffum affumere, quanta arterix eft capacitas: \& illumt ita fectioni indere, ut fuperior canalis pars altuus in arterix cauitatem pertingat, quàm fectionis fuperior fedes: \& ita inferior quog; canalis pars, dcorfum magis ipfa inferiori fectionis parte protrudatur, ac dein uinculum arteriæ circundetur, quod ipfrus corpus fuper canalem ftringat. Quum cnimid fit, fanguis qui $\mathrm{dem} \&$ fpiritus per arteriam a d pedem ufop excurrit, uerùm tota arterix pars canali fubdita non amplius pulfat. Soluto autem uinculo arterix pars canali fubdita, non minus quam fuperior per vis á corde perarteriascerebro deducatur pofteauidebimus: Ificesp.: pulfum L.,F. nunc Galeni uiuam in hac demonftratione confectionem fummopere mirantes, qua omnia m $\rightarrow$ ti. P,P præfcindi fuadet qux cerebro \& cordi funt cômunia, femper omittens' arterias, qux per tranf

c-Q.Q. uerfos uertcbrarû ceruicis proceffus caput petunt, \& ctiam nô mediocrem portionem uitalis

Th. T.T. fpiritus in " primos dura membranx finus, atç ita etiam in ccrebrum deferunt, adcò ut nô mi

Abb. $1 \Delta$ Auszug aus dem 7. Buch der Fabrica, in der Experimente mit Pulswellen beschrieben werden [2]

der Menge und Art von eingeworbenen Geldern. Aber auch Auftragsstudien für die Industrie oder Teilnahme an klinischen Studien können helfen, Gehälter für Mitarbeiter einzuwerben, und so Forschung am Laufen zu halten.

Mancher wird sich fragen, ob es sinnvoll ist, Geld in Forschung zu investieren. Hierzu habe ich eine klare Meinung, und dies bereits im Editorial von Heft 1/2019 für die vielen Gelder, die in die ISS investiert wurden [3], in Form eines Zitats zum Ausdruck gebracht:

Research that yields understanding of disease mechanisms and that can be applied to prevent or definitively treat disease will always prove to be cost-effective, even in a health system where incentives both for treatment and research may sometimes be less than optimal. [4]

Aber natürlich forschen wir insbesondere auch für unsere Patienten oder auch, um $\mathrm{zu}$ verhindern, dass wir Patienten werden: Die aktuellen Bemühungen zur Suche nach einem Impfstoff oder Behandlungsoptionen bei COVID-19 sind ein Beispiel. Der Nutzen für den Patienten mag bei der Grundlagenforschung oft nicht direkt sichtbar sein, aber auf diesem Wege wird die Basis für eine bessere $\mathrm{Pa}$ tientenversorgung gelegt: Neue verbesserte Bildgebung oder genetische Marker helfen bei der Diagnostik. Krankheiten werden früher und besser und vielleicht auch mit weniger Strahlung erkannt. Die Therapieergebnisse werden besser - neue, minimal-invasive Techniken senken die Morbidität und Mortalität. Neue Medikamente erlauben andere Therapieformen. Anhand von Markern wird die Behandlung auf den einzelnen Patienten zugeschnitten („tailored approach"). Forschung kann im Bereich der Prävention helfen, aber auch die Lebensqualität verbessern. Es gibt also unzählige Ansätze, mit denen wir unseren Patienten dank fortgesetzter Forschung helfen können.

Nun wissen Sie also, was unsere Autoren angetrieben hat. A. Gombert und M. Hakimi stimmen Sie dazu auch noch weiter ein.

\section{I) Natürlich forschen wir insbesondere auch für unsere Patienten}

Aber neben Artikeln zur Grundlagenforschung finden Sie in der vorliegenden Ausgabe eine Originalie (klinische Forschung: M. Schmitz: Chronische Nierenkrankheit und PAVK), Teil 2 zur akuten Karotis aus der Rubrik medizinische Evidenz (Literaturforschung: R. Grundmann), einen interessanten Fall (F. Meyer), eine Übersicht aus dem Netzwerk Grundlagenforschung (J.D. Rembe) und einen CME-Artikel zu den Zugangswegen (M. Steinbauer) sowie eine Arbeit zur Geschichte der Gefäßchirurgie (historische Forschung: W. Hach).
Also, diesmal liegt eine forschungslastige Ausgabe auf Ihrem Schreibtisch, PC oder mobilem Endgerät. Warum forschen wir also? Um ein gutes Leitthemenheft zu stellen.

Ich wünsche Ihnen viele neue Erkenntnisse und Spaß bei der Lektüre.

Herzlichst

Axel herra-Mvellawde

Prof. Dr. Axel Larena-Avellaneda

\section{Korrespondenzadresse

Prof. Dr. A. Larena-
Avellaneda
Klinik und Poliklinik
für Gefäßmedizin,
Universitäres Herz- und
Gefäßzentrum Hamburg,
Universitätsklinikum
Hamburg-Eppendorf
Martinistraße 52, Gebäude
Ost 70, 20246 Hamburg,
Deutschland
larena@uke.de

Interessenkonflikt. A. Larena-Avellaneda gibt an, dass kein Interessenkonflikt besteht.

\section{Literatur}

1. https://www.spektrum.de/lexikon/psychologie/ neugier/10472.Zugegriffen: 30.05 .2020

2. Vesalius: Fabrica, Libri septem, 1543, Basel

3. Larena-Avellaneda A (2019) Dank, Stabwechsel und ein paar Gedanken. Gefässchirurgie 24:5-6

4. Siegler M, Weisfeld A, Cronin D (2003) Is medical research cost effective? Response to Murphy and Topel. Perspect Biol Med 46(3):129-137 (Review. PubMed PMID: 14563079) 